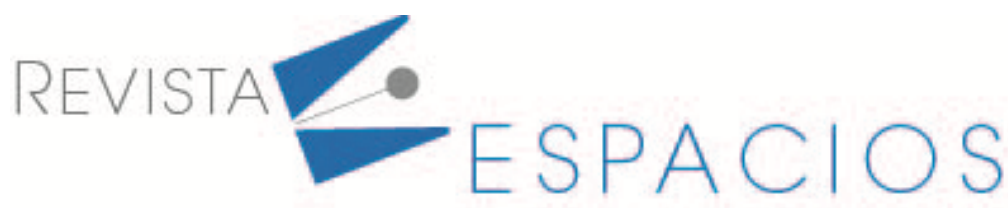

Vol. 41 (45) $2020 \cdot$ Art. 2

\title{
Las instituciones en América Latina y su incidencia en la productividad
}

\section{Institutions in Latin America and their incidence in productivity}

PINEDO, Jhon W. ${ }^{1}$

QUIROGA-PARRA, Darío ${ }^{2}$

TORRENT-SELLENS, Joan ${ }^{3}$

LORA, Carmen ${ }^{4}$

\section{Resumen}

El objetivo principal de este artículo es presentar una aproximación teórica y de estadística descriptiva del comportamiento de las instituciones y su incidencia en la productividad en veintidós (22) países de América Latina, puntualmente en los años 2000, 2014 y 2019. La metodología utilizada es la técnica documental para la realización del estado del arte en el contexto Latinoamericano y las técnicas de clúster y correlaciones para el análisis empírico. Los resultados muestran que los clústeres que agrupan a los países, no presentan importantes niveles de diferenciación.

Palabras clave: Instituciones; productividad; América Latina

\begin{abstract}
The main objective of this article is to present a theoretical and descriptive statistical approach to the behavior of institutions and their impact on productivity in twenty-two (22) Latin American countries, specifically in the years 2000, 2014 and 2019. The methodology used is the documentary technique for the realization of the state of the art in the Latin American context and the cluster and correlation techniques for empirical analysis. The results show that the clusters that group the countries do not present significant levels of differentiation
\end{abstract}

Key words: Institutions; productivity; Latin America

\section{Introducción}

Hasta la década de los años ochenta del siglo XX, el crecimiento económico en Latinoamérica presenta un comportamiento relativamente bajo al compararse con el de otras regiones del mundo. Este desempeño deficiente se asocia a la baja tasa de formación bruta de capital fijo en los países, fundamentalmente en tecnologías de información y comunicación, lo cual no permite un incremento sostenido de la productividad en varios de los países de la región (Aravena \& Hofman, 2014). Sin embargo, a partir del año 1990, Chile, Argentina,

\footnotetext{
${ }^{1}$ Economista y Ph.D. en Urbanismo. Investigador Facultad de Ciencias Administrativas de la Universidad Cooperativa de Colombia. Correo: jhon.pinedol@campusucc.edu.co

2 Ph.D. en sociedad de la información y el conocimiento profesor-Investigador Facultad de Ciencias Económicas de la Universidad Cooperativa de Colombia. dario.quirogap@campusucc.edu.co

${ }^{3} \mathrm{Ph}$. D. en sociedad de la información y el conocimiento. Catedrático de Economía en la Facultad de Economía de la Universitat Oberta de Catalunya. jtorrent@uoc.edu

${ }^{4}$ Abogada Constitucionalista. Investigadora Facultad de Derecho de la Universidad Cooperativa de Colombia. carmen.lora@campusucc.edu.co
} 
Costa Rica y Uruguay, logran crecimiento a una tasa per cápita por encima del promedio mundial, como consecuencia de una mayor tasa de acumulación de capital en sectores claves de la economía, que se traduce en una mayor productividad (Ros, 2014).

El crecimiento de la productividad a una tasa per cápita por encima del promedio mundial en Chile, Argentina, Costa Rica y Uruguay a partir de 1990, más la representativa participación de Brasil y México, son casos excepcionales en Latinoamérica que expresan la incorporación de mayor inversión en tecnologías y en mano de obra calificada. Esto dado que, la tendencia mostrada en la región es la persistencia de bajos niveles de productividad promedio y asimetrías notables entre los países mencionados y los de menor productividad como Nicaragua, Honduras, Bolivia, Guatemala y El Salvador, entre otros (Quiroga-Parra \& Torrent Sellens; 2015; OECD et al., 2019).

Es significativo anotar que, las instituciones juegan un rol importante en los niveles de productividad y en el crecimiento y desarrollo económicos de los países, incluidos los de América Latina. No obstante, los estudios empíricos sobre estas en el conexto latinoamericano se muestran escasos, dada la dificultad de obtener datos estadísticos.

En este trabajo, adicional al análisis teórico, se busca lograr una aproximación empírica desde la estadística, recurriendo a datos del Banco Mundial, fuente importante de información, que contribuye significativamente a superar las dificultades que enfrentan este tipo de investigaciones. El estudio empírico se realiza mediante análisis transversales, que ayudan a comprender el comportamiento de las instituciones en períodos diferentes, así como su mejoramiento o deterioro.

Este artículo tiene como propósito presentar una aproximación teórica y empírica, desde la perspectiva de la estadística descriptiva, acerca del comportamiento de las instituciones en veintidós países de América Latina y su incidencia en la productividad en los años 2000, 2014 y 2019. Lo anterior a partir del análisis estadístico de las tres variables institucionales establecidas: i) "Gasto público en educación” en \% del PIB; ii) "Gasto público en salud" en \% del PIB; iii) "Grado de corrupción" (expresada en percepción). El procesamiento estadístico se realizó con el software Stata. El propósito se logra mediante la realización de un estado del arte para Latinoamérica, así como de los análisis transversales, longitudinales y de correlaciones con la productividad, a partir de la información estadística obtenida del Banco Mundial.

El artículo inicia con los fundamentos teóricos, que incluyen estado del arte en América Latina; en el siguiente apartado se presenta la metodología, que permitió lograr el objetivo del trabajo; posteriormente se muestran los resultados obtenidos; seguidamente, se propone la discusión académica, y finalmente, son presentadas las principales conclusiones.

\subsection{Fundamentos teóricos y estado del arte}

En este trabajo el concepto de Instituciones es mucho más que el de organizaciones constituidas con el propósito de limitar las interacciones y crear orden en la sociedad (North, 1990). Las instituciones son organizaciones políticas, económicas y sociales determinantes para el bienestar general de una nación, la creación de empresas, innovación, el desarrollo tecnológico, la generación de riquezas y confianza entre todos los actores de la sociedad. Es decir, son organizaciones públicas y privadas que funcionan a través de mecanismos normativos, jurídicos y socioculturales (Kato \& Cárdenas, 2013).

Por su parte, la productividad es definida como la relación entre la producción y los recursos utilizados para obtenerla (Prokopenko, 1989). En el contexto macroeconómico de un país, su cálculo se obtiene al dividir el valor total de los bienes y servicios producidos sobre toda la población económicamente activa. Su crecimiento es explicado desde diferentes enfoques, dentro de los cuales es oportuno destacar el del crecimiento endógeno, 
que considera el aumento de la productividad como resultado del incremento del producto, la acumulación del capital físico y el progreso técnico (Young, 1928; Kaldor, 1957). Bajo esta perspectiva, el incremento de la productividad está asociado a la eficiencia y eficacia con que se utilizan los recursos en el proceso de producción (Medina, 2007).

Acemoglu, Johnson \& Robinson (2001) buscan proponer una teoría sobre las diferencias institucionales entre los países colonizados por los europeos, y se empeñan en establecer las causas fundamentales de las grandes diferencias en el ingreso per cápita entre los países. Señalan que las instituciones coloniales persistieron, incluso después de la independencia, y que las élites heredan instituciones extractivas, las cuales prefieren mantener para evitar incurrir en los costos de introducir mejores instituciones. Las "instituciones extractivas son aquellas que tienen por objetivo extraer rentas y riqueza de un subconjunto de la sociedad para beneficiar a un subconjunto distinto" (Acemoglu \& Robinson, 2012: 98).

Por su parte, Rodrik, Subramanian \& Trebbi (2002) destacan la importancia de la calidad institucional y su efecto significativo en la integración y en el desarrollo económico. A su vez, Glaeser, La Porta, López-de-Silanes, \& Shleifer (2004) reaniman el debate sobre si las instituciones políticas son causales de crecimiento económico, o si por el contrario, es el crecimiento el que conduce a la mejora de las instituciones.

Ahora bien, las instituciones de los países de América Latina se han caracterizado por su capacidad para superar situaciones complejas en los escenarios social, político y económico, originadas en períodos anteriores a la independencia política de sus Estados y persistentes después de dicha independencia, a través de la inestabilidad en los gobiernos y alteración del orden público, que trae como consecuencia el rezago tecnológico y económico de la región.

Este escenario lo describe en Colombia Kalmanovitz (2000), quien sostiene con determinación que las instituciones se encuentran influidas, entre otras, por ideologías ilegales y religiosas, que conducen en circunstancias a la debilidad institucional, falta de autoridad para hacer cumplir las leyes y corrupción. Considera que, las instituciones deben garantizar los derechos de propiedad de los productores e impedir que sus excedentes o recursos sean capturados por otros agentes, ya sea por medio de acciones de sanción social o a través de medidas de fuerza.

Por otro lado, Gutiérrez \& Rodríguez (2003) formulan una reflexión sobre la relación crecimiento-desarrollo, desde la perspectiva de los cambios institucionales en el siglo XX. En este sentido, es pertinente destacar que la prosperidad económica de una nación requiere de instituciones que promuevan una mayor inversión en actividades productivas y un mejor marco de intercambio (Oriol, 2007).

En un significativo trabajo empírico, Ros (2014) destaca las experiencias de crecimiento a partir del año 1990 en la región de América Latina, y la expansión de Chile, Argentina, Costa Rica y Uruguay a una tasa per cápita por encima del promedio mundial. Así mismo, resalta que Colombia, México, Brasil, Ecuador, Guatemala, República Bolivariana de Venezuela, Paraguay, El Salvador, Honduras, Bolivia y Nicaragua lo hicieron a una tasa menor. Señala también que, en el período de 1952 a 1980, el mejor desempeño de la productividad se presentó en Brasil, con $4.58 \%$ anual, seguido de México, con 3.15\%, Ecuador, con 3.02\%, y Chile, con $2.47 \%$, mientras que los países de menor desempeño fueron Bolivia, con $1.59 \%$, Venezuela, con $1.88 \%$ y Colombia, con $1.99 \%$.

En una línea similar, Aravena \& Hofman (2014) analizan el crecimiento económico, la productividad y sus determinantes en cinco países principales de América Latina (Argentina, Brasil, Chile, Colombia y México), durante el período 1990-2010. Este análisis se aplica para el agregado de la economía y para nueve sectores económicos, y utilizan la base de datos LA-KLEMS como herramienta fundamental que muestra las tendencias comparativas de productividad. Estos dos últimos trabajos, por sus aportes empíricos, contribuyen 
positivamente al estado del arte sobre el tema de la productividad en los países latinoamericanos y estimularon el desarrollo del presente trabajo.

\section{Metodología}

El estudio siguió metodológicamente, en todas sus partes el concepto, científico universal del discurso del método de Descartes (1637) el cual se sintetiza en sus cuatro principios: i) Principio de duda o evidencia sistemática; ii) Principio del análisis o descomposición; iii) Principio de la síntesis o la composición; iv) Principio de la enumeración o de la verificación. En donde, el sistema cartesiano ha sido de especial importancia en el uso de la presente técnica y el de Kant (1787) en todo su contexto.

La población objeto de estudio o universo corresponde a 32 países de América Latina, de donde se tomaron 22 países como muestra, esto es, el 68.8\%. El número de países seleccionados estuvo sujeto a la restricción de los datos disponibles en la fuente de datos internacional del Banco Mundial. Los 22 países seleccionados fueron: Argentina (ARG), Bolivia (BOL), Chile ( $\mathrm{CHL}$ ), Colombia ( $\mathrm{COL})$, Panamá (PAN), República Dominicana (DOM), República Bolivariana de Venezuela (VEN), Costa Rica (CRI), Ecuador (ECU), El Salvador (SLV), Guatemala (GTM), Honduras (HND), Nicaragua (NIC), Paraguay (PRY), Brasil (BRA), Cuba (CUB), Guyana (GUY), Jamaica (JAM), México (MEX), Perú (PER), Trinidad y Tobago (TTO) y Uruguay (URY).

El trabajo empírico, desde la estadística descriptiva, estuvo fundamentado en la metodología estadística de análisis multivalente, partiendo de la técnica de factoriales, en específico, con la técnica de clúster en un sistema cartesiano. Dicha técnica fue seleccionada como conglomerados aleatorizados no jerárquicos, homogéneos a nivel intra-grupal y heterogéneo a nivel intergrupal, mediante el método de las k-medias. A la par, se tomaron los períodos transversales 2000, 2014 y 2019.

El análisis clúster tomó como objetivo clasificar $\rho$ objetos en $k$ grupos mediante el uso de $n$ variables; en este sentido, los elementos a clasificar son los 22 países de América Latina en 3 grupos, usando 3 variables: "Gasto público en educación" (\% del PIB), "Gasto público en salud" (\% del PIB), y Grado de corrupción (nivel de percepción), asociadas al concepto de Instituciones. Variables consideradas críticas, y que afectan los procesos de crecimiento y desarrollo de los países, a través de los niveles de productividad y eficiencia (Quiroga-Parra \& Torrent Sellens, 2015).

La técnica matemática, que parte del uso de factoriales para llegar a los clústeres, correspondió a algoritmos iterativos que facilitan clasificar estos mediante el método de las k-medias, haciendo uso de las distancias euclidianas existentes a nivel intragrupo, considerando el conjunto de variables cuantitativas involucradas. La optimización se logra maximizando el grado de homogeneidad de los individuos seleccionados en cada conglomerado. De hecho, se puede señalar que los ítems agrupados en cada conjunto son similares dentro de la perspectiva de la variable involucrada en el proceso (Hair, Anderson, Tatham \& Black, 2008). La parte empírica del estudio, manejó matemáticamente la técnica de correlaciones (Gujarati \& Porter, 2010), para conocer en la fase de hechos de productividad, el posible nivel de relación entre la productividad y las instituciones, considerando las variables más relevantes de esta. En el trabajo se toma el PIB per cápita como una medida de productividad (Jorgenson, 2001), acorde con la literatura internacional, en específico, el planteamiento de la función de producción lineal:

$Y_{i, t}=A_{i, t} f\left(K_{i, t}, L_{i, t}\right)$

Donde $Y_{i, t}$ corresponde al output agregado del país $i$ en el perído $t$. De su parte $A_{i, t}$ en términos de la teóría neoclásica, es un factor neutral que incrementa el volumen agregado de los inputs, y que acá en términos de la teoría endógena es llamada tecnología o conocimiento del país $i$, en el período $t$. A su vez, $K_{i, t}$ son los inputs del capital, y $L_{i, t}$ son los inputs del trabajo del país $i$, en el período $t$. De manera específica, en términos del Hick- 
neutral de Jorgenson (2001) y de la teoría endógena, en $A_{i, t}$ se encuentra las variables de instituciones que inciden en la productividad. La ecuación [1] al divididirla por la población nos representa el PIB per cápita. La interpretación de las correlaciones, en este caso, se ha realizado tanto gráfica como matemáticamente.

\section{Resultados}

\subsection{Clúster instituciones para América Latina en el año 2000}

Es oportuno iniciar expresando que estos resultados se presentan mediante tablas, gráficos y texto. En este orden, la tabla 1 presenta, en sus nueve columnas, los resultados recabados sobre las instituciones en veintidós países de América Latina. Las tres primeras columnas muestran el agrupamiento de los países estudiados en forma de clústeres; la columna número cuatro, de izquierda a derecha, muestra las variables institucionales estudiadas, mientras que las restantes revelan los datos del comportamiento de los clústeres objeto de estudio.

Tabla 1

Resumen clúster instituciones para AL, año 2000

\begin{tabular}{|c|c|c|c|c|c|c|c|c|}
\hline \multicolumn{3}{|c|}{ Clúster } & \multirow{2}{*}{$\begin{array}{c}\text { Variables } \\
\text { Instituciones }\end{array}$} & \multicolumn{2}{|c|}{ ANOVA } & \multicolumn{3}{|c|}{ Estadísticos Descriptivos } \\
\hline 1 & 2 & 3 & & $\mathbf{F}$ & Sig & Clúster 1 & Clúster 2 & Clúster 3 \\
\hline \multirow[t]{3}{*}{ PRY } & \multirow{3}{*}{$\begin{array}{c}\text { ARG, BOL, } \\
\text { CRI, ECU, } \\
\text { GTM, HND, } \\
\text { MEX, NIC, } \\
\text { PAN, SLV, } \\
\text { VEN }\end{array}$} & \multirow{3}{*}{$\begin{array}{l}\text { BRA, CHL, } \\
\text { COL, CUB, } \\
\text { GUY, JAM, } \\
\text { PER, DOM, } \\
\text { TTO, URY }\end{array}$} & $\begin{array}{c}\text { Gasto público en } \\
\text { educación (\% del PIB) }\end{array}$ & 0.299 & 0.745 & $\begin{array}{c}\bar{x}=4,571 \\
\sigma=\cdots\end{array}$ & $\begin{array}{l}\bar{x}=3,726 \\
\sigma=1,341\end{array}$ & $\begin{array}{l}\bar{x}=4,280 \\
\sigma=2,193\end{array}$ \\
\hline & & & $\begin{array}{l}\text { Gasto público en salud } \\
\text { (\% del PIB) }\end{array}$ & 0.084 & 0.920 & $\begin{array}{c}\bar{x}=3,231 \\
\sigma=\cdots\end{array}$ & $\begin{array}{l}\bar{x}=3,409 \\
\sigma=1,418\end{array}$ & $\begin{array}{l}\bar{x}=3,641 \\
\sigma=1,525\end{array}$ \\
\hline & & & $\begin{array}{l}\text { Grado de corrupción } \\
\text { (nivel de percepción) }\end{array}$ & 97.55 & 0.000 & $\begin{array}{c}\bar{x}=86,007 \\
\sigma=\cdots\end{array}$ & $\begin{array}{c}\bar{x}=23,389 \\
\sigma=6.875\end{array}$ & $\begin{array}{l}\bar{x}=7,306 \\
\sigma=3,625\end{array}$ \\
\hline
\end{tabular}

Fuente: cálculos propios con base en información del Banco Mundial (2020)

Para el año 2000, el clúster 1 está conformado solamente por Paraguay. El clúster 2 lo integran: Argentina, Bolivia, Costa Rica, Ecuador, Guatemala, Honduras, México, Nicaragua, Panamá Salvador y Venezuela. El número 3 lo integran: Brasil, Chile, Colombia, Cuba, Guyana, Jamaica, Perú, República Dominicana, Trinidad y Tobago y Uruguay. El agrupamiento de mayor valor en medias, en sus variables de las Instituciones para el año 2000, es el número 1 , y el de promedios más bajos es el número 3. Los p-valores del estadístico de la prueba $\mathrm{F}$ expresan que los tres agrupamientos por instituciones solo se diferencian por la variable "Grado de corrupción (nivel de percepción)", la cual posee un nivel de significancia de 0,000, señalando que, los clústeres se encuentran diferenciados entre si y significativamente diferentes solo respecto a los componentes de esta última variable.De este modo, el clúster 1 presenta una media de 4,57 para la variable "Gasto público en educación" (\% del PIB), un promedio de 3,23 para la variable "Gasto público en salud" (\% del PIB), y una media de 86 en la variable "Grado de corrupción" (\% de empresas con pagos no oficiales) observándose que el clúster 1 es el que presenta el mayor nivel de corrupción de la región, representado, en este caso, únicamente por Paraguay; no obstante, este clúster muestra los promedios más altos en gastos de educación y salud.

En el clúster número 2, se observa un promedio de 3,726 de "Gasto público en educación" (\% del PIB), una media de 3,40 en la variable "Gasto público en salud" (\% del PIB), y un promedio de 23,39 en la variable "Grado de corrupción" (percepción) como \% de empresas con pagos no oficiales. Dicho clúster ostenta el segundo promedio más alto de niveles de corrupción, en concreto con el país El Salvador. De su parte, en el clúster número 3 el promedio de "Gasto público en educación" (\% del PIB) es de 4,28, así mismo el Gasto público en salud (\% del PIB) es de 3,64 en su promedio y en la variable Grado de corrupción (nivel de percepción) se observa una media de 7,31. De manera que el clúster número 3 es el que presenta menor nivel de corrupción en el año 2000, en los países de Cuba y República Dominicana. En este orden de ideas, desde la perspectiva de los menores niveles de corrupción y de los mejores aportes a Gastos de educación y de salud, como porcentaje del PIB, el clúster 3 se 
muestra como el de superior comportamiento en términos de calidad en sus instituciones en América Latina; esto es de instituciones más inclusivas. De manera opuesta, y con la misma perspectiva, el clúster 1 , especialmente por sus altos niveles de corrupción se muestra como el de menor calidad de las instituciones, que en términos de Acemoglu (2012) se revelan con instituciones extractivas.

Así mismo, en el gráfico 1, el panel "a" muestra la relación entre el "Gasto público en educación" (\% del PIB) versus el "Gasto público en salud" (\% del PIB). Uruguay, Costa Rica y Cuba muestran los mejores valores en Gastos público en educación (\% del PIB). Por otro lado, de manera opuesta, se observa a Ecuador, Trinidad Tobago y Guatemala, seguidos de República Dominicana, México y Venezuela, como los países de menor gasto en salud en la región. El mismo panel expone que los gastos públicos en educación (\% del PIB) son liderados, en el año 2000, por Guyana y Cuba, seguidos de lejos por países como Paraguay, Bolivia, Jamaica y Panamá. Por su parte, Colombia se muestra por debajo de la media de todos los países de la región. En la parte inferior de este tipo de gasto se muestran Ecuador, República Dominicana, Trinidad Tobago, Salvador y Uruguay.

\section{Gráfico 1}

Clústeres de instituciones para AL, año 2000

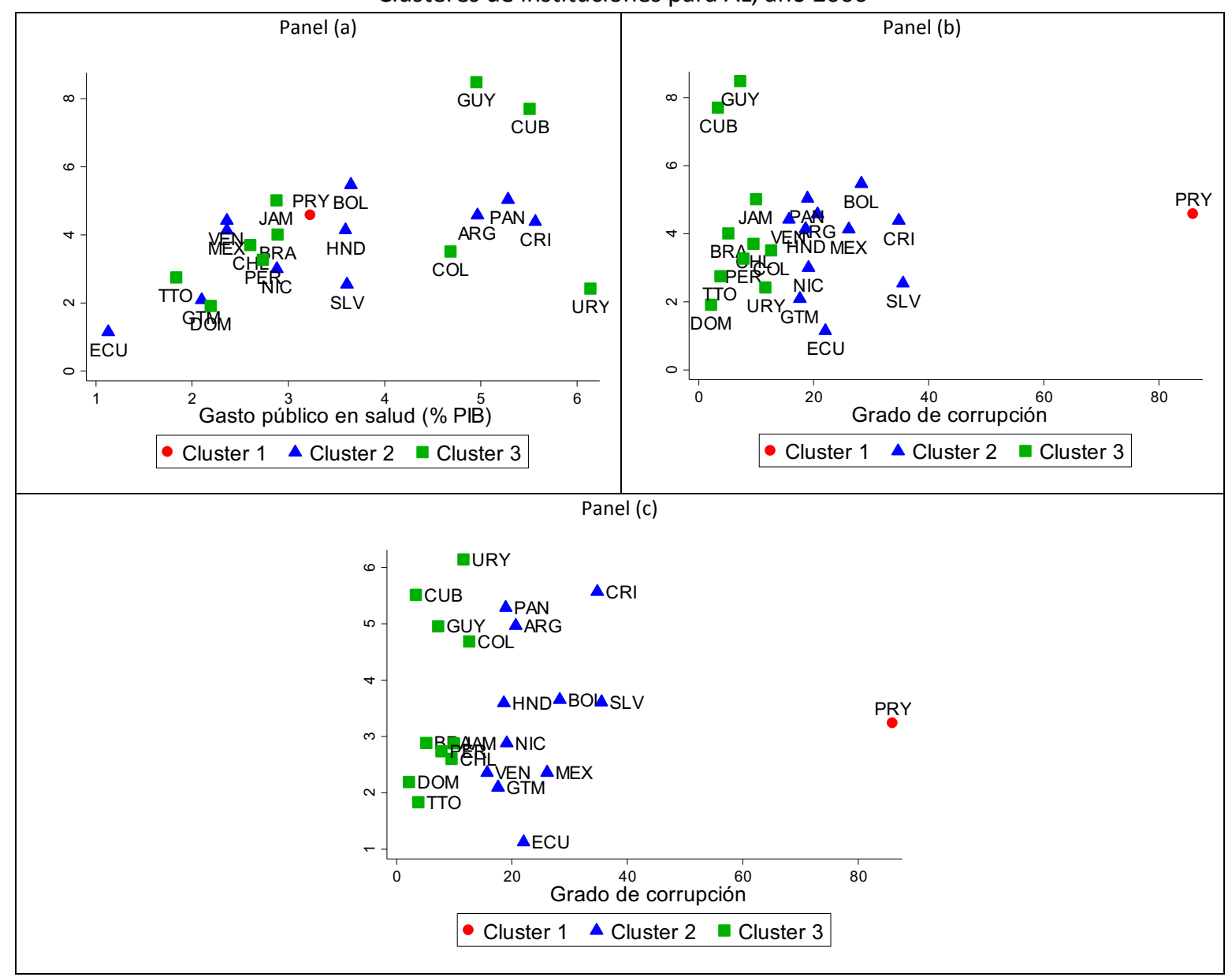

Fuente: cálculos propios con base en información del Banco Mundial (2020)

El panel "b", que contrasta el "Grado de corrupción" versus el "Gasto público en educación", resalta que el país de mayores niveles de corrupción es Paraguay, seguido por El Salvador. Los países de menor nivel de corrupción muestran ser República Dominicana y Cuba. La mayor parte de los países de la región se muestran por debajo del nivel medio de la escala de 0-100. El gráfico del panel reafirma los esfuerzos por invertir en educación que hacen Guyana y Cuba en el año 2000. 
El panel "c" del mismo gráfico contrasta el "Gasto en salud" versus el "nivel de corrupción". En el se evidencia que Uruguay y Cuba, seguidos de cerca por Costa Rica, denotan esfuerzos por mejorar los niveles de educación en el período del año 2000. Del análisis transversal realizado en este gráfico, puede señalarse también a Ecuador como el país de menor inversión en la educación.

\subsection{Clúster instituciones para América Latina, año 2014}

La tabla 2 presenta un resumen de los clústeres de América Latina para el año 2014. El clúster 1 está formado únicamente por Panamá. El conglomerado 2 lo integran Argentina, Bolivia y Perú. El número 3 lo conforman dieciocho países: Brasil, Chile, Colombia, Costa Rica, Cuba, República Dominicana, Ecuador, Guatemala, Guyana, Honduras, Jamaica, México, Nicaragua, Paraguay, Salvador, Trinidad y Tobago, Uruguay y Venezuela.

Tabla 2

Resumen clúster instituciones para AL, año 2014

\begin{tabular}{|c|c|c|c|c|c|c|c|c|}
\hline \multicolumn{3}{|c|}{ Clúster } & \multirow{2}{*}{$\begin{array}{l}\text { Variables } \\
\text { Instituciones }\end{array}$} & \multirow{2}{*}{$\begin{array}{l}\text { ANOVA } \\
\text { F }\end{array}$} & \multirow{2}{*}{$\begin{array}{l}\text { Sig } \\
\end{array}$} & \multicolumn{3}{|c|}{ Estadísticos Descriptivos } \\
\hline 1 & 2 & 3 & & & & Clúster 1 & Clúster 2 & Clúster 3 \\
\hline \multirow[t]{3}{*}{ PAN } & $\begin{array}{l}\text { ARG } \\
\text { BOL } \\
\text { PER }\end{array}$ & $\begin{array}{c}\text { BRA, CHL, COL, } \\
\text { CRI, CUB, } \\
\text { DOM, ECU, } \\
\text { GTM, GUY, }\end{array}$ & $\begin{array}{l}\text { Gasto público en } \\
\text { educación (\% del PIB) }\end{array}$ & 0.564 & 0.578 & $\begin{array}{c}\bar{x}=3.225 \\
\sigma=\cdots\end{array}$ & $\begin{array}{l}\bar{x}=5.217 \\
\sigma=1.454\end{array}$ & $\begin{aligned} \bar{x} & =5.601 \\
\sigma & =2.285\end{aligned}$ \\
\hline & & $\begin{array}{l}\text { MEX, NIC, PRY, } \\
\text { SLV, TTO, URY, } \\
\text { VEN }\end{array}$ & $\begin{array}{l}\text { Gasto público en salud } \\
\text { (\% del PIB) }\end{array}$ & 0.027 & 0.973 & $\begin{array}{c}\bar{x}=4.891 \\
\sigma=\cdots\end{array}$ & $\begin{array}{l}\bar{x}=4.515 \\
\sigma=1.401\end{array}$ & $\begin{array}{l}\bar{x}=4.422 \\
\sigma=2.077\end{array}$ \\
\hline & & & $\begin{array}{l}\text { Grado de corrupción } \\
\text { (Percepción) }\end{array}$ & 71.652 & 0.000 & $\begin{array}{l}\bar{x} \\
=36.181 \\
\quad \sigma=\cdots\end{array}$ & $\begin{array}{c}\bar{x}=20.097 \\
\sigma=3.462\end{array}$ & $\begin{array}{l}\bar{x}=7.621 \\
\sigma=2.654\end{array}$ \\
\hline
\end{tabular}

Fuente: cálculos propios con base en información del Banco Mundial (2020)

De los tres clústeres, los de mayor "Gasto público en educación" (como \% del PIB) son, según orden de mayor a menor, el clúster 3, seguido del número 2 y finalmente el clúster 1, con valores promedio de 5,60, 5,21 y 3,22, respectivamente. Los "Gastos públicos en salud" (como \% del PIB) son liderados por el clúster 1, seguido del clúster 2, y finalmente, el clúster 3, con valores promedio de 4,89; 4,51 y 4,42, en su orden. Finalmente, los "Grado de corrupción" (percepción) se muestran en mayor grado en el clúster 1, con 36,18 de percepción señalando a Panamá como el país con mayores niveles de corrupción en el año 2014. Le siguen en orden el clúster 2 , con una media de 3,46, y el clúster 3 , con 2, 65 .

En el gráfico 2, se presenta en el panel "a" la relación "Gasto público en salud" versus "Gasto público en educación", ambos como \% del PIB; observándose en el año 2014 a Cuba como el país de mayor inversión en educación, seguido de Costa Rica y de los demás países de la región. Por otra parte, Guatemala y Trinidad Tobago se muestran como los países con el menor gasto en educación. A su vez, Colombia se muestra en un punto medio bajo en su atención a la educación. 
Gráfico 2

Clústeres de instituciones para AL, año 2014

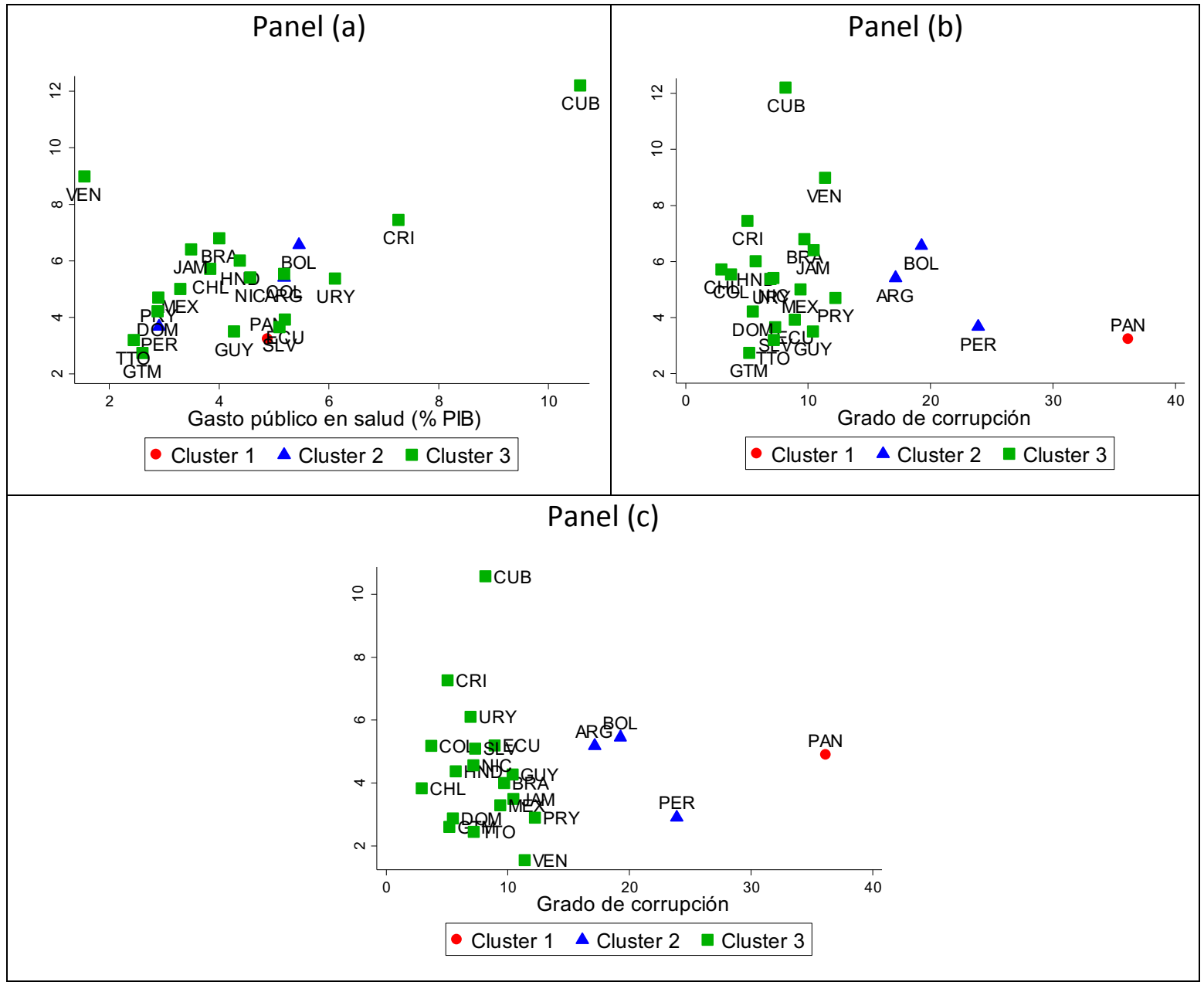

Fuente: cálculos propios con base en información del Banco Mundial (2020)

En el mismo gráfico, en el panel "b" se contrasta el "Gasto público en educación" versus el "Grado de corrupción" (percepción), mostrándose que el país que ostenta los mayores niveles de corrupción en el año 2014 es Panamá, mientras que el de menores niveles es Chile. En el panel "c" se analiza el "Gasto público en salud" y el "grado de corrupción", en donde Cuba y Costa Rica muestran resultados favorables.

\subsection{Clúster instituciones para América Latina, año 2019}

Un análisis de clústeres para el año 2019 se presenta en la tabla 3. Esta muestra la agrupación de los veintidós países de América Latina con esta técnica. En la primera sección de la tabla muestra al clúster 1 conformado por Argentina, Colombia, Cuba, Guyana y Perú. El clúster 2 lo integran Chile, Costa Rica, Jamaica, Trinidad y Tobago y Uruguay. El tercero lo componen Bolivia, Brasil Ecuador, Salvador, Guatemala, Honduras, México, Nicaragua, Panamá, Paraguay, República Dominicana y Venezuela. Clústeres agruapdos alrededor de las variables de Instiuciones como "Grado de corrupción" (Índice de Percepción), "Gastos del gobierno en educación" como \% del PIB, y "Gastos del gobierno en salud" como \% del PIB.

En la segunda parte de la tabla está la Anova, en donde se denotan que, el p-valor de 0,000 del estadístico F de la avariable "Grado de corrupción" (percepción) contibuye solamente por esta variable a la diferenciación de los tres clústeres, haciendo que estos sean significativamente diferentes entre si. 
Tabla 3

Resumen clúster instituciones para AL, año 2019

\begin{tabular}{|c|c|c|c|c|c|c|c|c|}
\hline \multicolumn{3}{|c|}{ Clúster } & \multirow{2}{*}{$\begin{array}{c}\text { Variables } \\
\text { Instituciones }\end{array}$} & \multicolumn{2}{|c|}{ ANOVA } & \multicolumn{3}{|c|}{ Estadísticos Descriptivos } \\
\hline 1 & 2 & 3 & & $\mathbf{F}$ & Sig & Clúster 1 & Clúster 2 & Clúster 3 \\
\hline \multirow[t]{3}{*}{$\begin{array}{l}\text { ARG, COL, } \\
\text { CUB, GUY, } \\
\text { PER }\end{array}$} & \multirow{3}{*}{$\begin{array}{l}\text { CHL, } \\
\text { CRI, } \\
\text { JAM, } \\
\text { TTO, } \\
\text { URY }\end{array}$} & \multirow{3}{*}{$\begin{array}{l}\text { BOL, BRA, } \\
\text { ECU, SLV, } \\
\text { GTM, HND, } \\
\text { MEX, NIC, } \\
\text { PAN, PRY, } \\
\text { DOM, VEN }\end{array}$} & $\begin{array}{l}\text { Grado de corrupción } \\
\text { (Índice de Percepción) }\end{array}$ & 89.658 & 0.000 & $\begin{array}{r}\bar{x}=43.71 \\
\sigma=4.46\end{array}$ & $\begin{aligned} \bar{x} & =62.08 \\
\sigma & =6.24\end{aligned}$ & $\begin{array}{r}\bar{x}=28.53 \\
\sigma=4.22\end{array}$ \\
\hline & & & $\begin{array}{l}\text { Gastos del gobierno en } \\
\text { educación como \% del } \\
\text { PIB }\end{array}$ & 0.414 & 0.667 & $\begin{array}{l}\bar{x}=6.67 \\
\sigma=4.97\end{array}$ & $\begin{aligned} \bar{x} & =5.523 \\
\sigma & =1.15\end{aligned}$ & $\begin{array}{l}\bar{x}=5.38 \\
\sigma=1.77\end{array}$ \\
\hline & & & $\begin{array}{l}\text { Gastos del gobierno en } \\
\text { salud como \% del PIB }\end{array}$ & 0.387 & 0.684 & $\begin{aligned} \bar{x} & =8.55 \\
\sigma & =4.91\end{aligned}$ & $\begin{array}{r}\bar{x}=6.977 \\
\sigma=2.44\end{array}$ & $\begin{aligned} \bar{x} & =7.507 \\
\sigma & =1.916\end{aligned}$ \\
\hline
\end{tabular}

Fuente: cálculos propios con base en información del Banco Mundial (2020)

En la parte tres de la tabla se observan los estadísticos descriptivos. Observándose que para la variable "Grado de corrupción" (Índice de Percepción), el clúster 2 presenta los mayores índices de corrupción, en donde se encuentran cinco países, con una media de percepción equivalente a 62,08. Seguido del clúster 1 y 3 . Al año 2019, los países con menores niveles de corrupón muestran ser Bolivia, Brasil Ecuador, Salvador, Guatemala y Honduras.

Los países que obstentan mayores nivles de "Gasto público en educación" se observan ubicados en el clúster 1, seguido del clúster 2 y el 3. A su vez, los países con mayores niveles en salud y educación se encuentran ubicados en el clúster 1, conformado por Argentina, Colombia, Cuba, Guyana y Perú. Países caracterizados por expresar niveles medios de corrrupcón (percepción).

En la misma dirección, el gráfico 3 presenta la conformación de los tres clústeres usando la técnica de dispersión. Conforme lo indica el p-valor del gráfico, los tres clústeres se observan poco diferenciados, Sin embargo, en el panel "a" y "b" de los tres clústeres se obssrvan más difrenciados, en especial los números 2 y 1 . La diferenciación de estos se detecta específicamente con la variable Grado de corrupción. La gráfica de dispersión permite detectar los mayores problemas en las instituciones, en especial a través de la variable niveles de corrupción. Resultados estos que van en la misma dirección de la literatura expuesta, en especial la de Acemoglu, Johnson \& Robinson (2001; 2012).

Así mismo, el panel "c" señala que los veintidós países estudiados de América Latina, ostentan bajos niveles de inversión en educación y salud como porcentaje del PIB y mayores promedios de los nivles de corrupción. Aspecto este que va en deterioro de la calidad de las instituciones, conforme lo expone la literatura internacional analizada. 
Gráfico 3

Clústeres de instituciones

para AL, año 2019

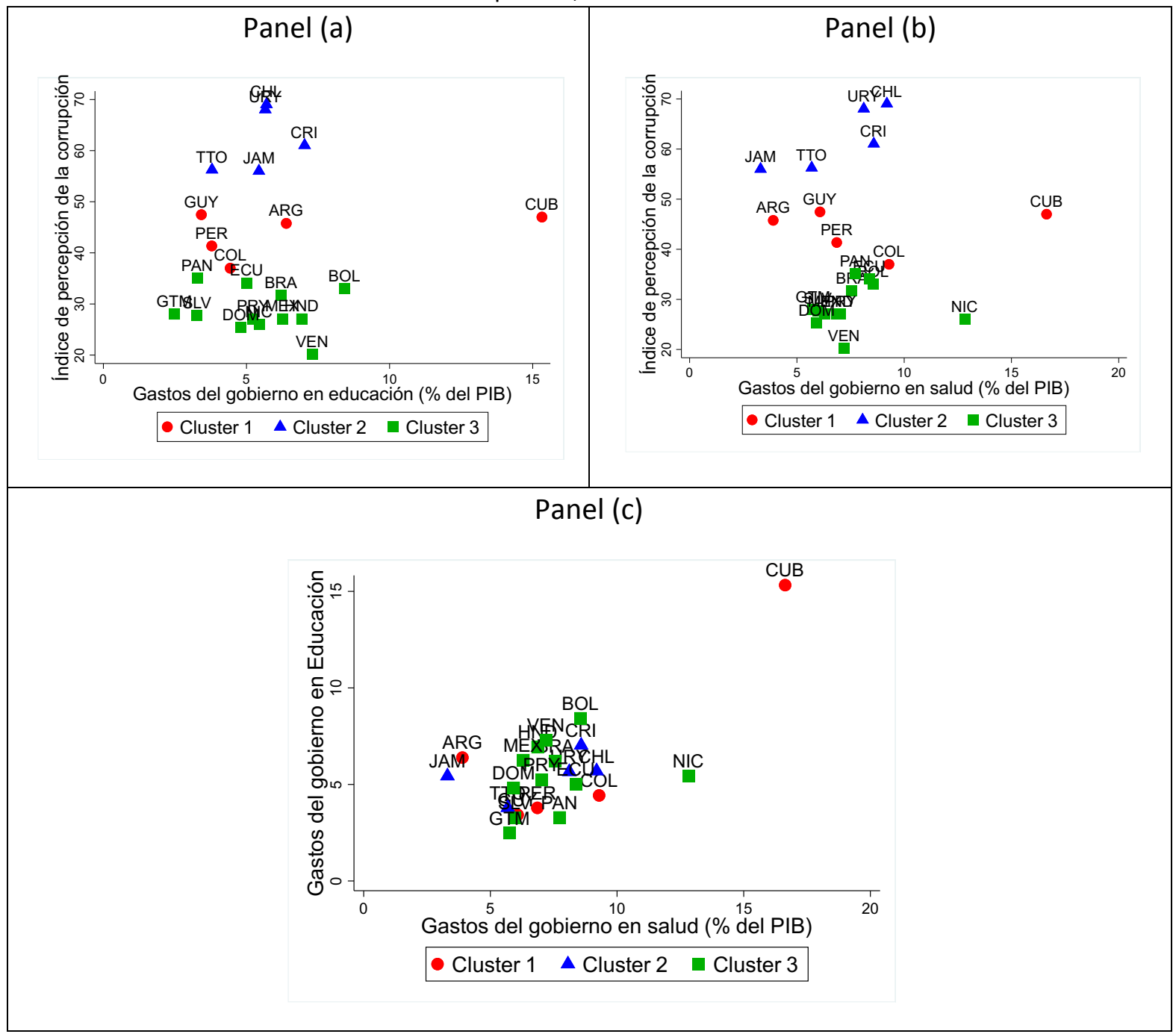

Fuente: cálculos propios con base en información del Banco Mundial.

En síntesis, los gráficos $1 ; 2$ y 3, así como los datos estadísticos respectivos exponen heterogeneidad en las inversiones en educación y salud. Los países con los mejores esfuerzos de "Gastos en educación" como \% del PIB, para el año 2000 son: Guyana, Cuba, Paraguay, Bolivia y Panamá; para el año 2014 Cuba, Venzuela, Costa Rica; y para el año 2019, Cuba y Bolivia. En "Gastos del gobierno en salud" como \% del PIB, los mejores esfuerzos se observan para el año 2000 en Uruguay, Cuba, Costa Rica, Panamá, Guyana, Argentina y Colombia en su orden; en el año 2014 Cuba, Venezuela, Costa Rica; y para el año 2019 Cuba, Nicaragua, Chile y Colombia.

En términos de "Grado de corrupción" (Índice de Percepción), en los períodos estudiados se observa: año 2000, Paraguay, Salvador; año 2014, Panamá, Perú, Bolivia, Argentina; año 2019, Chile, Uruguay, Costa Rica, Trinidad Tobago y Jamaica. Por tanto, las decisiones y las políticas de las instituciones de América Latina en las inversiones en educación y salud pueden ser reflejados en los resultados de productividad y niveles de ingreso per cápíta de la población, que son analizados en los siguientes apartados. 


\subsection{Correlaciones entre Productividad y variables de Instituciones}

El presente apartado busca conocer la posible existencia de correlación entre el PIB per cápita, asimilado este como una medida de productividad (Jorgenson, 2001), y las diferentes variables de instituciones, estudiadas en las secciones anteriores. En el gráfico 4 se presenta el comportamiento de la correlación entre PIB per cápita y el "Gasto público en educación" (\% del PIB) para los 22 países estudiados, tomando el período de los años 2000 al 2019. El gráfico muestra la importante correlación entre estas dos variables, con un comportamiento positivo para 19 países, excepto para Guyana, Jamaica y Panamá, de los cuales 16 países exponen correlación positiva y sigificativas, esto es el $73 \%$ de los países. Así mismo, todas las correlaciones muestran un p-valor entre 0,000 y 0,005 , significativas en el nivel 0,01 (bilateral).

Gráfico 4

Correlaciones entre el PIB per-cápita y Gasto público en educación (\% del PIB) para países de AL, 2000-2019

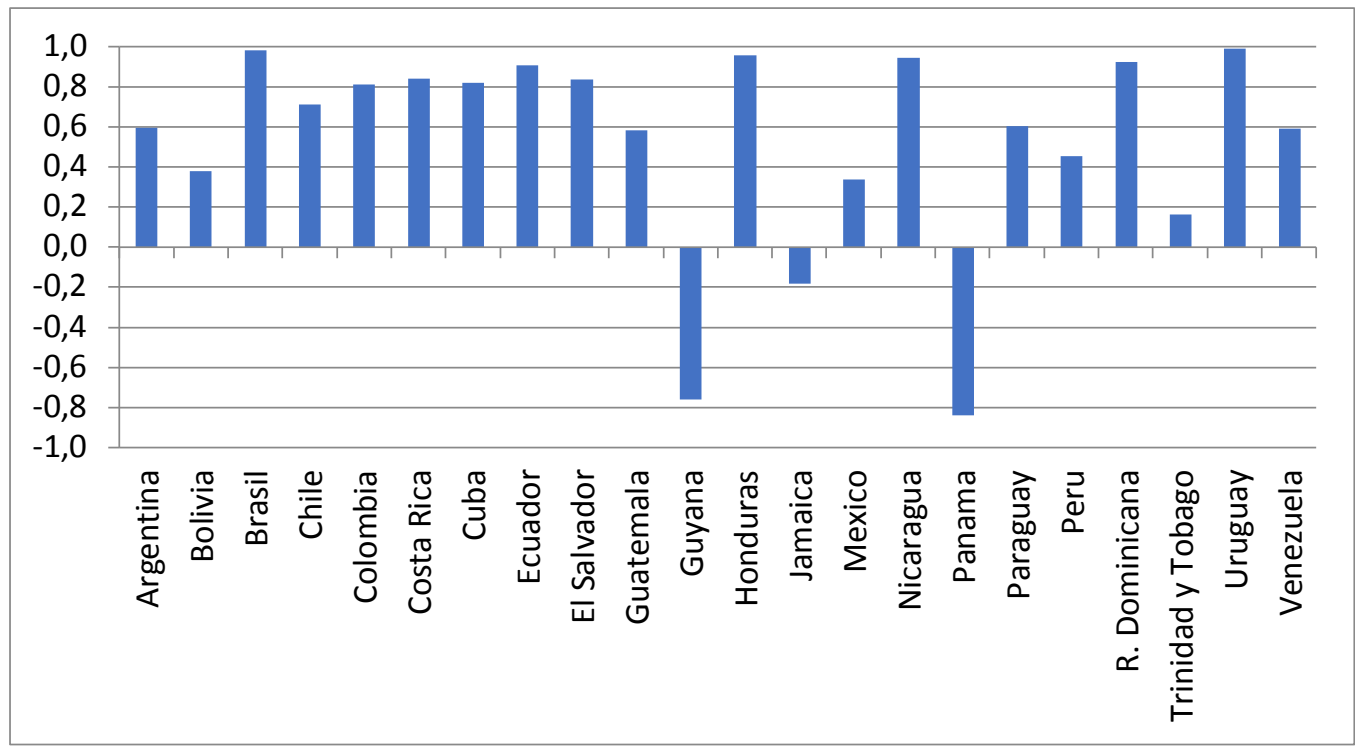

Fuente: cálculos propios con base en información del Banco Mundial

Los países con mayores grados de correlación de la variable "Gasto público en educación" (\% del PIB) con la productividad mostraron ser: Brasil, Honduras, Nicaragua, República Dominicana y Uruguay, países que presentan los mayores promedios de Gasto en educación, ubicados en el clúster 3 de la tabla 2 como el mejor promedio de gasto en educación, aspecto observado igualmente para Brasil, República Dominicana y Uruguay en el año 2000 (tabla 1). Para el año 2019, en el clúster 3 el promedio descendió, con exepción de Uruguay que pasó al clúster 2. Con fundamento en lo anterior, hipotéticamente, a priori (Kant, 1787) se puede intuir que, a mayores aportes a la educación, mayores grados de correlación se observan con la productividad.

Por su parte, el Gráfico 5 muestra las correlaciones entre el PIB per-cápita versus el "Gasto público en salud" (\% del PIB) para los 22 países de AL, en el período 2000-2019. Dieciocho de los veintidós países presentan una correlación positiva. Los países con correlación negativa son: Guyana, Jamaica, Panamá y Venezuela. Las salidas del software Stata señalan que 12 de los 22 países presentan correlación positiva y significativa y con p-valor inferior al 0,005. Los países de correlación positiva y significativa son: Bolivia, Brasil, Chile, Colombia, Costa Rica, Cuba, Ecuador, El Salvador, México, Nicaragua, República Dominicana y Uruguay. Para el año 2000, los anteriores países muestran promedios de gastos importantes en salud (tabla 1). 


\section{Gráfico 5}

Correlaciones entre el PIB per-cápita, Gasto público en salud (\% del PIB) para países de AL, 2000-2019

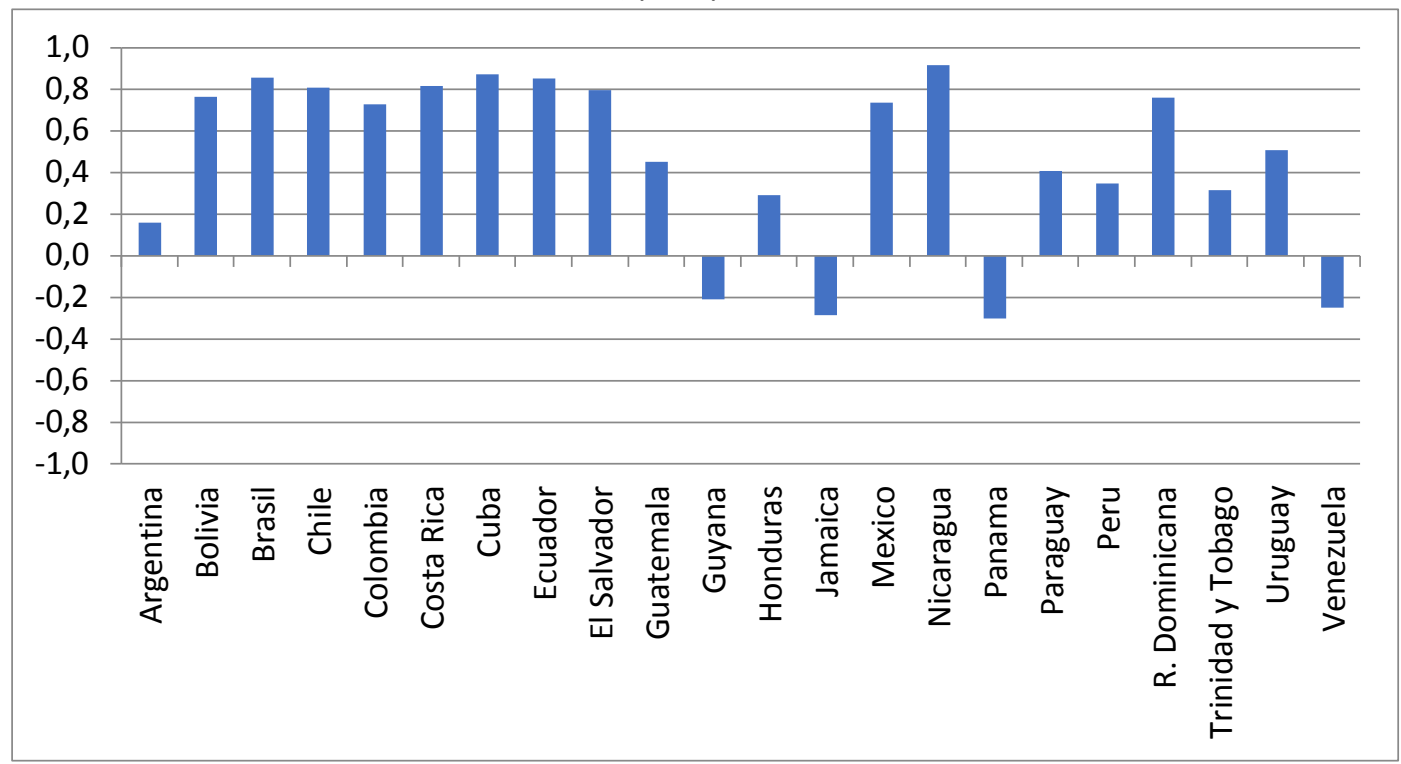

Fuente: cálculos propios con base en información del Banco Mundial

El "Gasto público en salud" (\% del PIB) presenta importantes niveles positivos de correlaclón con la productividad para el $82 \%$ de los países estudiados y el $55 \%$ muestran correlación positiva y significativa. De donde se intuye que estos gastos están contribuyendo a mejorar la productividad. Los países de correlación no significativa entre las variables PIB per-cápita versus el "Gasto público en salud" (\% del PIB) son: Argentina, Guatemala, Guyana, Honduras, Jamaica, Panamá, Paraguay, Perú, Trinidad Tobago y Venezuela. Estos países, para el año 2000 muestran gastos de salud medio bajos y niveles de corrupción medios altos (ver tabla 1 y gráfico 1). Para el año 2014 los mismos países presentan gastos de salud bajos. Así mismo, el gráfico 2 expresa que, al menos siete de los diez países señalados exhiben niveles de corrupción por encima del promedio estadístico de sus similares, o alrededor de este, de donde se infiere que los bajos gastos de salud en un país y el "Grado de corrupción" afectan la correlación con los niveles de productividad. Para el año 2019, los anteriores países mejoraron sus promedios de "Gasto público en salud" (\% delPIB)

Por otra parte, el gráfico 6 presenta las correlaciones entre el PIB per-cápita y el "Grado de corrupción" (percepción) para países de América Latina entre 2000-2019. El gráfico expone que siete de los países presentan correlación positiva entre las dos variables estudiadas, y en su defecto, los restantes países tienen correlación negativa. Igualmente, se observa que de los 22 países estudiados, Guyana, Jamaica y Venezuela muestran correlación positiva no significativa, y exhiben valores cercanos a cero, con p-valor por encima del 0,005. Siete países muestran correlación positiva significativa y un p-valor inferior a 0,005: Trinidad Tobago, República Dominicana, Cuba, Brasil, Panamá y Perú, mientras que, de los 13 países de correlación negativa, todos muestran ser significativa, con p-valor inferior a 0,005. 


\section{Gráfico 6}

Correlaciones entre el grado de corrupción y

el PIB per-cápita para países de AL, 2000-2019

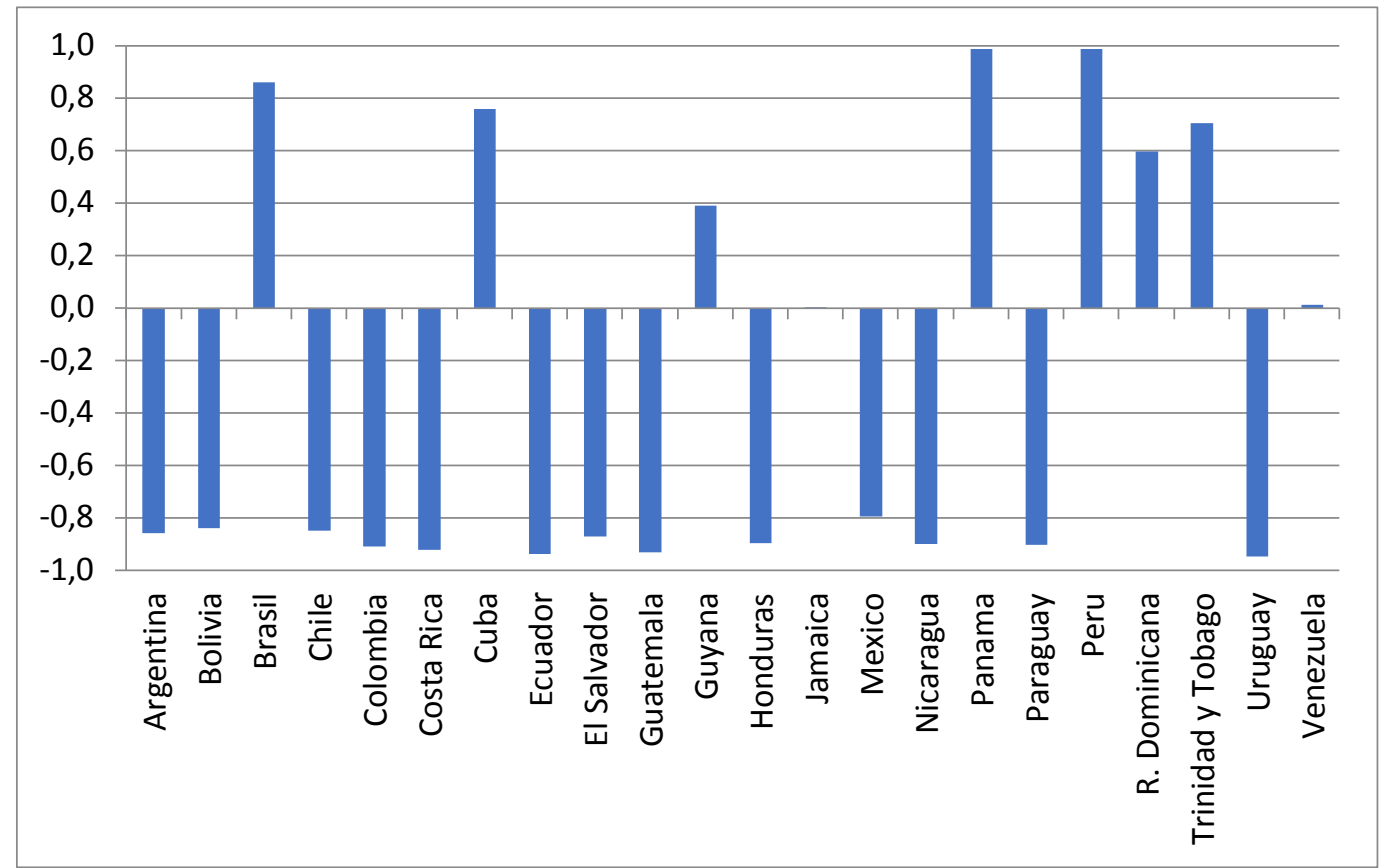

Fuente: cálculos propios con base en información del Banco Mundial

En síntesis, de los veintidós países de América Latina solamente tres de ellos no muestran tener correlación significativas entre PIB per cápita y "Gastos de salud" (\% del PIB), eson son: Venezuela, Jamaica y Guyana. Los trece restantes muestran correlación negativa y significativa y seis de ellos correlación positiva igualmente significativa.

Los resultados expresan de manera longitudinal en el período 2000-2019, así como transversalmente en el primero y último período estudiado, que los gastos de educación y de salud no son relevantes, mientras que los niveles de corrupción se observan elevados. Los bajos gastos en educación y salud, al igual que el alto nivel de percepción sobre la corrupción, muestran estar afectando la productividad en América Latina. En este orden, los resultados expresan fuertes coincidencias teóricas que reflejan instituciones poco eficientes en buena parte de los países de América Latina. Finalmente, los datos obtenidos pueden considerarse como primeros hallazgos de investigación, que sería recomendable verificarlos posteriormente con pruebas econométricas.

\section{Conclusiones}

El presente trabajo realiza aportes importantes a la literatura internacional, especialmente a la de América Latina, sobre el tema de Instituciones y su incidencia en la productividad. En primer término, se hace una primera aproximación académica sobre la literatura de trabajos existentes sobre instituciones en la región. Segundo, se realiza un análisis sobre las tres variables más relevantes que pueden estar afectando la productividad y el crecimiento económico de los países, variables controladas esencialmente por las instituciones de cada país. Tercero, los conglomerados (clústeres), y los pares de variables utilizadas, así como la técnica de correlaciones, permitieron corroborar la importancia de las instituciones y el efecto que estas tienen sobre la productividad y la calidad de vida en estos países.

El estudio facilitó analizar, en un período de 20 años, el comportamiento de las variables de Gastos en educación, Gastos en salud y Grado de corrupción, arrojando resultados que son necesarios para que los tomadores de 
decisiones de los países puedan diseñar políticas apropiadas, que contribuyan al mejoramiento de la productividad y del crecimiento económico y sostenido.

Los clústeres no presentan estadísticamente un importante nivel de diferenciación entre ellos. De manera que, los gastos de los gobiernos se observan heterogéneos en el período analizado, sin ningún tipo de tendencia, con excepción de unos pocos países.

El análisis estadístico y las percepciones a priori producto de las técnicas utilzadas intuyen que, a mayores aportes a la educación, mayores grados de correlación positiva con la productividad. Los resultados indican que, los "Gasto público en educación" (\% del PIB) presentan importantes niveles positivos de correlaclón con la productividad para el $82 \%$ de los países estudiados, y el 55\% muestran correlación positiva y significativa. De donde se intuye que estos gastos están contribuyendo a mejorar la productividad.

Se evidencia que, los bajos gastos de salud en América Latina y los elevados niveles de percepción sobre la corrupción afectan de manera importante la correlación con los niveles de productividad. Así mismo, se observa que los países con mayor inversión en educación presentan menores niveles de percepción de corrupciòn. La técnica de correlaciones contribuyó a mostrar la relevancia que tienen las instituciones sobre los niveles de productividad de los países estudiados.

Finalmente, se corrobora la necesidad de profundizar en el tema de las instituciones y su incidencia en la productividad y de realizar futuros estudios en esta línea de investigación, con el propósito de medir la eficiencia de las instituciones de los países de América Latina y su contribución a la productividad.

\section{Referencias bibliográficas}

Acemoglu, D. \& Robinson, J. (2012). Por qué fracasan los países. Barcelona: Planeta.

Acemoglu, D., Johnson, S. \& Robinson, J. (2001). The Colonial Origins of Comparative Development: An Empirical Investigation. The American Economic Review, 91 (5): 1369-1401. Consultado 25 agosto 2020: http://www.jstor.org/stable/2677930.

Aravena, C. \& Hofman, A. (2014). Crecimiento económico y productividad en América Latina. Santiago de Chile: CEPAL y Naciones Unidas.

Banco Mundial. (2020). PIB y PIB PER CÁPITA 1960-2019. Recuperado de http://datos.bancomundial.org/indicador/NY.GDP.MKTP.CD

Descartes R. (1637). El discurso del método. Traducción de Risieri Frondizi. 1999. Madrid: Alianza.

Glaeser, E., La Porta, R., López-de-Silanes, F. \& Shleifer, A. (2004). Do institutions cause growth. Working Paper 10568. Disponible en: http://www.nber.org/papers/w10568.

Gutiérrez, L. \& Rodríguez, F. (2003). Historia, instituciones y crecimiento económico. Análisis Económico, 18, (38): 97-122.

Gujarati, D., \& Porter, D. (2010). Econometría. México: Mc Graw Hill.

Hair, J., Anderson, R., Tatham, R. \& Black, W. (2008). Análisis multivariante. Madrid: Pearson Educación S.A. Jorgenson, D. (2001). Information technology and the U.S. economy. American Economic Review, 9 (1): 1-32. Kaldor, N. (1957). A model of economic growth. Economic Journal, 67, 591-624. 
Kalmanovitz, S. (2000). Las instituciones colombianas en el siglo XX. Disponible en: http://www.banrep.gov.co/docum/ftp/borra131.pdf.

Kant, I. (1787). Crítica de la razón pura. Traducción de José del Perojo, 1984. Bogotá: Ediciones Universales.

Kato, E. \& Cárdenas, C. (2013). Instituciones, transición demográfica y riesgos del sistema de pensiones. Norteamérica, 2: 105-126.

Medina, J. (2007). Modelo Integral de productividad. Bogotá: Universidad Sergio Arboleda.

North, D. (1990). Instituciones, cambio institucional y desempeño económico. Disponible en: www.cepal.org/ilpes/noticias/paginas/4/15434/north90.doc.

OCDE et al. (2019), Perspectivas económicas de América Latina 2019: Desarrollo en transición, OECD. Publishing, Paris, https://doi.org/10.1787/g2g9ff1a-es.

Oriol, J. (2007). Revisión crítica de los aportes del institucionalismo a la teoría y a la práctica del desarrollo. Revista de Economía Institucional, 9 (16): 121-148.

Prokopenko, J. (1989). Gestión de la productividad. Ginerbra: Organización Internacional del Trabajo.

Quiroga-Parra, D. (2013). TIC, conocimiento, innovación y productividad. Un análisis empírico comparado sobre las fuentes de la eficiencia en América Latina, países asiáticos y OECD. Tesis Doctoral, Universidad Oberta de Catalunya, Barcelona.

Quiroga-Parra, D.J. \& Torrent-Sellens, J. (2015). Las nuevas fuentes de productividad en América Latina y la OCDE [online working paper]. (Working Paper Series; WP14-009). IN3 Working Paper Series. IN3 (UOC), pp. 1-34. 2015. ISSN: 2013- 8644. URL: http://journals.uoc.edu/ojs/ index.php/in3-working-paperseries/article/ view/n14-quiroga-parra-torrent-sellens/ n14-quiroga-parra-torrent-sellens.

Rodrik, D., Subramanian, A. \& Trebbi, F. (2002). Institutions rule: the primacy of institutions over geography and integration in economic development". Working Paper 9305. Disponible en: http://www.nber.org/papers/w9305

Ros, J. (2014). Productividad y crecimiento en América Latina: ¿por qué la productividad crece más en unas economías que en otras? México. CEPAL \& ONU.

Young, A. (1928). Increasing returns and economic progress. Economic Journal, 38 (152): 527-542.

Esta obra está bajo una Licencia Creative Commons Attribución-NoCommercial 4.0 International

(cc) EY-NO 